\title{
Twenty-five-year trajectories of insulin resistance and pancreatic $\beta$-cell response and diabetes risk in nonalcoholic fatty liver disease
}

\author{
Lisa B. VanWagner ${ }^{1,2}$ (D) | Hongyan Ning ${ }^{2}$ | Norrina B. Allen ${ }^{2}$ | Juned Siddique ${ }^{2}$ | \\ April P. Carson $^{3}$ | Michael P. Bancks ${ }^{2}$ | Cora E. Lewis ${ }^{4}$ | John Jeffrey Carr ${ }^{5}$ | \\ Elizabeth Speliotes $^{6}$ | Norah A. Terrault ${ }^{7}$ | Mary E. Rinella ${ }^{1}$ | Miriam B. Vos ${ }^{8}$ (D) | \\ Donald M. Lloyd-Jones ${ }^{2,9}$
}

\footnotetext{
${ }^{1}$ Division of Gastroenterology \& Hepatology, Northwestern University, Chicago, IL, USA

${ }^{2}$ Department of Preventive Medicine, Northwestern University, Chicago, IL, USA

${ }^{3}$ Department of Epidemiology, University of Alabama at Birmingham, Birmingham, AL, USA

${ }^{4}$ Division of Preventive Medicine, University of Alabama at Birmingham, Birmingham, AL, USA

${ }^{5}$ Department of Radiology, Vanderbilt University, Nashville, TN, USA

${ }^{6}$ Division of Gastroenterology, University of Michigan, Ann Arbor, MI, USA

${ }^{7}$ Division of Gastroenterology, University of California at San Francisco, San Francisco, CA, USA

${ }^{8}$ Division of Gastroenterology, Department of Pediatrics, Emory University, Atlanta, GA, USA

${ }^{9}$ Division of Cardiology, Northwestern University, Chicago, IL, USA
}

\section{Correspondence}

Lisa B. VanWagner, Division of Gastroenterology \& Hepatology and Department of Preventive Medicine, Northwestern University, Chicago, IL, USA.

Email: Ivw@northwestern.edu

Funding information

The CARDIA Study is supported by contracts HHSN268201300025C,

HHSN268201300026C,

HHSN268201300027C,

HHSN268201300028C,

HHSN268201300029C,

HHSN268200900041C and AG0005 from the National Institutes of Health (NIH). Drs. VanWagner (KL2TR001424), Bancks (T32HL069771) and Carr (R01HL098445) are supported by the $\mathrm{NIH}$.

Handling Editor: Luca Valenti

\begin{abstract}
Background \& Aims: Insulin resistance is a risk marker for non-alcoholic fatty liver disease, and a risk factor for liver disease progression. We assessed temporal trajectories of insulin resistance and $\beta$-cell response to serum glucose concentration throughout adulthood and their association with diabetes risk in non-alcoholic fatty liver disease.

Methods: Three thousand and sixty participants from Coronary Artery Risk Development in Young Adults, a prospective bi-racial cohort of adults age 1830 years at baseline (1985-1986; YO) who completed up to 5 exams over 25 years and had fasting insulin and glucose measurement were included. At Y25 (2010-2011), non-alcoholic fatty liver disease was assessed by noncontrast computed tomography after exclusion of other liver fat causes. Latent mixture modelling identified 25-year trajectories in homeostatic model assessment insulin resistance and $\beta$-cell response homeostatic model assessment- $\beta$.

Results: Three distinct trajectories were identified, separately, for homeostatic model assessment insulin resistance (low-stable [47\%]; moderate-increasing [42\%]; and high-increasing [12\%]) and homeostatic model assessment- $\beta$ (low-decreasing
\end{abstract}

Abbreviations: BMI, body mass index; CARDIA, Coronary Artery Risk Development in Young Adults; Cl, confidence interval; CT, computed tomography; EU, exercise units; FPG, fasting plasma glucose; FPI, fasting plasma insulin; HDL-C, high density lipoprotein cholesterol; HOMA, homeostatic model assessment; HU, Hounsfield units; IR, insulin resistance; LA, liver attenuation; NAFLD, non-alcoholic fatty liver disease; OR, odds ratio; SBP, systolic blood pressure; T2DM, type 2 diabetes mellitus; Y0, year 0. 
[16\%]; moderate-decreasing [63\%]; and high-decreasing [21\%]). Y25 non-alcoholic fatty liver disease prevalence was $24.5 \%$. Among non-alcoholic fatty liver disease, high-increasing homeostatic model assessment insulin resistance (referent: lowstable) was associated with greater prevalent (OR 95\% Cl = 8.0, 2.0-31.9) and incident $(\mathrm{OR}=10.5,2.6-32.8)$ diabetes after multivariable adjustment including Y0 or Y25 homeostatic model assessment insulin resistance. In contrast, non-alcoholic fatty liver disease participants with low-decreasing homeostatic model assessment- $\beta$ (referent: high-decreasing) had the highest odds of prevalent ( $O R=14.1,3.9-50.9)$ and incident (OR = 10.3, 2.7-39.3) diabetes.

Conclusion: Trajectories of insulin resistance and $\beta$-cell response during young and middle adulthood are robustly associated with diabetes risk in non-alcoholic fatty liver disease. Thus, how persons with non-alcoholic fatty liver disease develop resistance to insulin provides important information about risk of diabetes in midlife above and beyond degree of insulin resistance at the time of non-alcoholic fatty liver disease assessment.

\section{KEYWORDS}

coronary artery risk development in young adults, non-alcoholic fatty liver disease, nonalcoholic steatohepatitis, obesity

\section{1 | INTRODUCTION}

Non-alcoholic fatty liver disease (NAFLD) is considered the hepatic manifestation of the metabolic syndrome with a well-established association with insulin resistance (IR). NAFLD prevalence approaches 70\% among persons with type 2 diabetes mellitus (T2DM). ${ }^{1}$ However, NAFLD also occurs among persons without T2DM and may precede the development of T2DM. ${ }^{2,3}$ Once established, T2DM promotes liver disease progression and is an independent risk factor for liver cancer. ${ }^{2}$ There are multiple potential common pathogenic mechanisms in NAFLD and T2DM. The cascade of IR leading to hyperinsulinaemia and then pancreatic $\beta$-cell dysfunction coupled with defective lipid metabolism and ensuing hepatic triglyceride accumulation are described in both NAFLD and T2DM. ${ }^{4}$ Thus, multiple studies have evaluated the role of insulin-sensitizing agents as treatment for NAFLD. ${ }^{5}$ However, improving IR alone does not appear sufficient to resolve NAFLD., One speculative explanation for these findings might be that despite improvement in insulin sensitivity, pancreatic $\beta$-cell function continues to deteriorate and thus targeting insulin sensitivity alone has a null effect. Recent data demonstrate that biopsy-proven NAFLD is associated with an exaggerated pancreatic $\beta$-cell response. ${ }^{8}$ However, clinical imaging-based NAFLD studies have failed to demonstrate a relationship between NAFLD and pancreatic $\beta$-cell dysfunction., ${ }^{9,10}$ There are a lack of population-level studies examining changes in pancreatic $\beta$-cell response to blood glucose concentration throughout adulthood in relation to changes in IR on NAFLD and T2DM.

Surrogate indicators for IR, as well as pancreatic $\beta$-cell response to glucose concentration, can be extrapolated from fasting

\section{Key Points}

- In NAFLD, presence of diabetes increases risk for liver disease progression.

- Early identification of risk factors for diabetes is an important strategy to improve outcomes in NAFLD.

- Increasing insulin resistance from young adulthood into middle age is associated with greatest risk of NAFLD and subsequent diabetes.

- Thus, how insulin resistance develops in NAFLD provides important information about risk of diabetes independent of degree of insulin resistance in middle age.

blood glucose and insulin levels that are commonly included in population-based studies. The homeostatic model assessments (HOMA) are simple methods for estimating $\beta$-cell response to glucose concentration and how well insulin is utilized by its target cell populations. ${ }^{11}$ Specifically, HOMA-IR is a measure for whole body IR and HOMA- $\beta$ is a measure of pancreatic $\beta$-cell response to glucose concentration. ${ }^{11}$ High HOMA-IR equates to high levels of tissue IR and high HOMA- $\beta$ equates to high $\beta$-cell response. We sought to characterize temporal trends in HOMA-IR and HOMA- $\beta$ during young adulthood in relation to prevalent NAFLD in middle adulthood and subsequent risk of T2DM among persons with NAFLD. 


\section{2 | METHODS}

\section{1 | Study sample}

The Coronary Artery Risk Development in Young Adults (CARDIA) study is an ongoing longitudinal cohort study that enrolled 5115 black and white men and women 18-30 years of age from 4 U.S. field centres. The baseline exam (1985-1986; year 0, Y0) and follow-up exams at 2, 5, 7, 10, 15, 20 and 25 years after baseline included extensive collection of detailed clinical data, including non-contrast computed tomography (CT) measurement of liver fat at Y25. Retention rates among survivors have been high throughout the study with $>90 \%$ of the surviving cohort maintaining contact. ${ }^{12}$ Participants provided written informed consent at each examination, and institutional review boards from each field centre (University of Alabama at Birmingham, Birmingham, AL; Northwestern University, Chicago, IL; University of Minnesota, Minneapolis, MN; and Kaiser Permanente, Oakland, CA) approved the study annually.

Fasting glucose and insulin were measured at Y0, Y7, Y10, Y15, Y20, and Y25. Measures at examinations when the participant was pregnant were excluded $(n=192)$. Measures at examinations when the participant reported taking exogenous insulin were excluded for calculation of HOMA- $\beta$ ( $n=29) .{ }^{11,13}$ Of 3060 participants with fasting measures at $\mathrm{YO}$ and at 3 or more follow-up examinations, 2455 had liver fat assessed at Y25. Participants were then excluded if they had a self-reported history of cirrhosis, hepatitis ( $n=38$ ), a risk factor for chronic liver disease (eg, intravenous drug use, $n=52$ ) or secondary hepatic steatosis: alcohol consumption $>14$ standard drinks/week in women and $>21$ standard drinks/week in men $(n=225)^{7}$ human immunodeficiency virus ( $n=14)$, and medications (eg, valproic acid, methotrexate, tamoxifen and/or amiodarone; $n=22$ ). The remaining 2104 participants formed the NAFLD-eligible sample population for the HOMA-IR analyses. For HOMA- $\beta$ analyses, the sample was 2089 because of 15 participants who had reported exogenous insulin use that resulted in less than three repeated fasting insulin measures over time (Figure S1).

\section{2 | Measurements}

Standardized protocols for data collection were used across study centres and have previously been described. ${ }^{12,14}$ Blood was drawn after a 12-hour fast in the seated position, separated and plasma frozen to $-70^{\circ} \mathrm{C}$ prior to analysis in a central laboratory. ${ }^{12}$ Glucose was assayed at YO with the hexokinase UV method by American Bio-Science Laboratories (Van Nuys, CA) and by hexokinase coupled to glucose-6-phosphate dehydrogenase (Merck Millipore, Billerica, MA) at Y7, Y10, Y15, Y20 and Y25. Insulin measurements were determined by radioimmunoassay (Linco Research, St. Charles, MO) at Y0, Y7, Y10, Y15 and Y20, as well as by an Elecsys sandwich immunoassay (Roche Diagnostics, Rotkreuz, Switzerland) at Y25. HOMA-IR, HOMA- $\beta$ were calculated as:
$\mathrm{HOMA}-\mathrm{IR}=(\mathrm{FPI} \times \mathrm{FPG}) / 22.5$,

HOMA- $\beta=(20 \times F P I) /(F P G-3.5)$,

where FPI is fasting plasma insulin concentration $(\mu \mathrm{U} / \mathrm{L})$ and FPG is fasting plasma glucose (mmol/L). ${ }^{11,13}$

The presence of T2DM was assessed at each examination based on a combination of medication use for T2DM (every examination), FPG $\geq 126 \mathrm{mg} / \mathrm{dL}$ (Y0, Y7, Y10, Y15, Y20 and Y25), 2-hour glucose $\geq 200 \mathrm{mg} / \mathrm{dL}$ (Y10, Y20 and Y25) by OGTT, or haemoglobin $A_{1 c}\left(H_{b A_{1 c}}\right) \geq 6.5 \%$ (Y20 and Y25). Prevalent T2DM was defined as meeting T2DM criteria at Y25 only and incident T2DM was defined as meeting T2DM criteria at any exam year $(0,7,10,15,20$ or 25), thus incidence is greater than prevalence.

The CT protocol included the heart and abdomen using a noncontrast CT scan performed using GE (GE 750HD 64 and GE LightSpeed VCT 64, Birmingham and Oakland Centers, respectively; GE Healthcare, Waukesha, WI) or Siemens (Sensation 64, Chicago and Minneapolis Centers; Siemens Medical Solutions, Erlangen, Germany) multidetector CT scanners and has been described previously. ${ }^{15}$ Quality control and image analysis was performed at a core reading centre (Wake Forest University Health Sciences, Winston-Salem, NC). Any (eg, Mild-Moderate-Severe) NAFLD was defined as liver attenuation (LA) $<51$ Hounsfield Units (HU, equivalent to a liver/spleen ratio

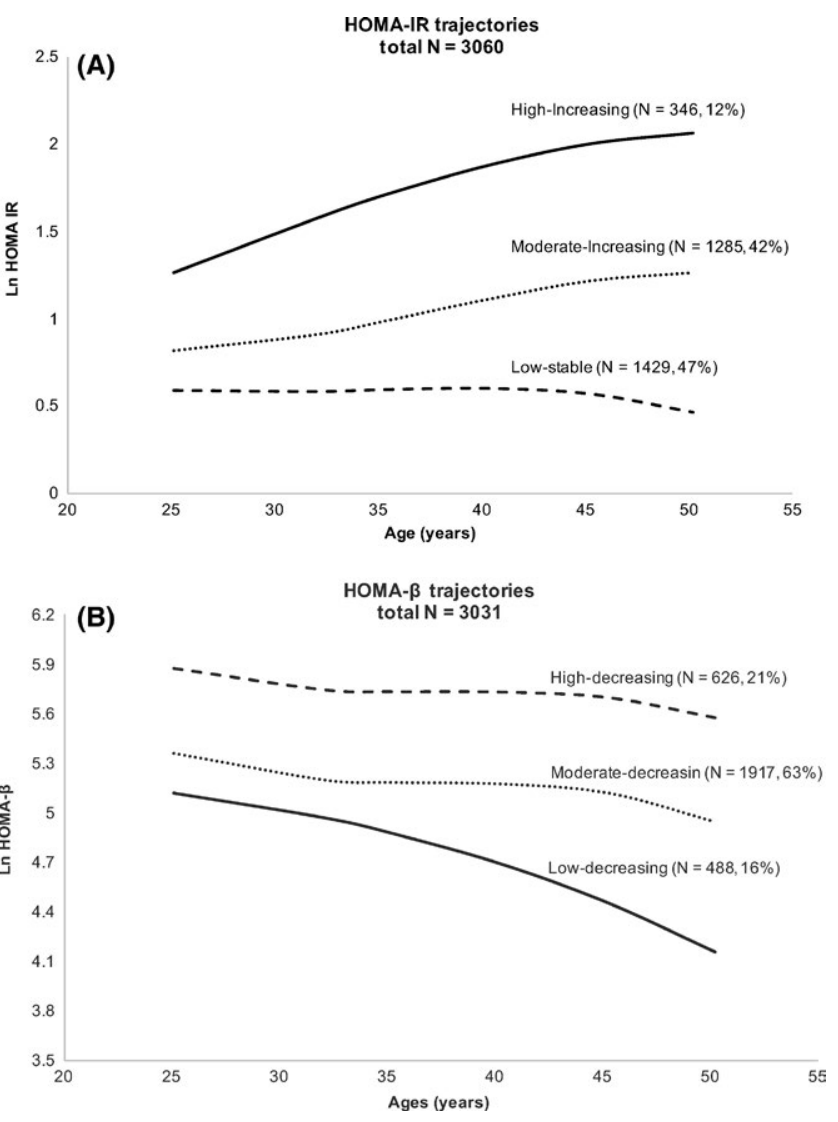

FIGURE 1 Trajectories by Age in the Coronary Artery Risk Development in Young Adults (CARDIA) Study of A, HOMA-IR and B, HOMA- $\beta$ 


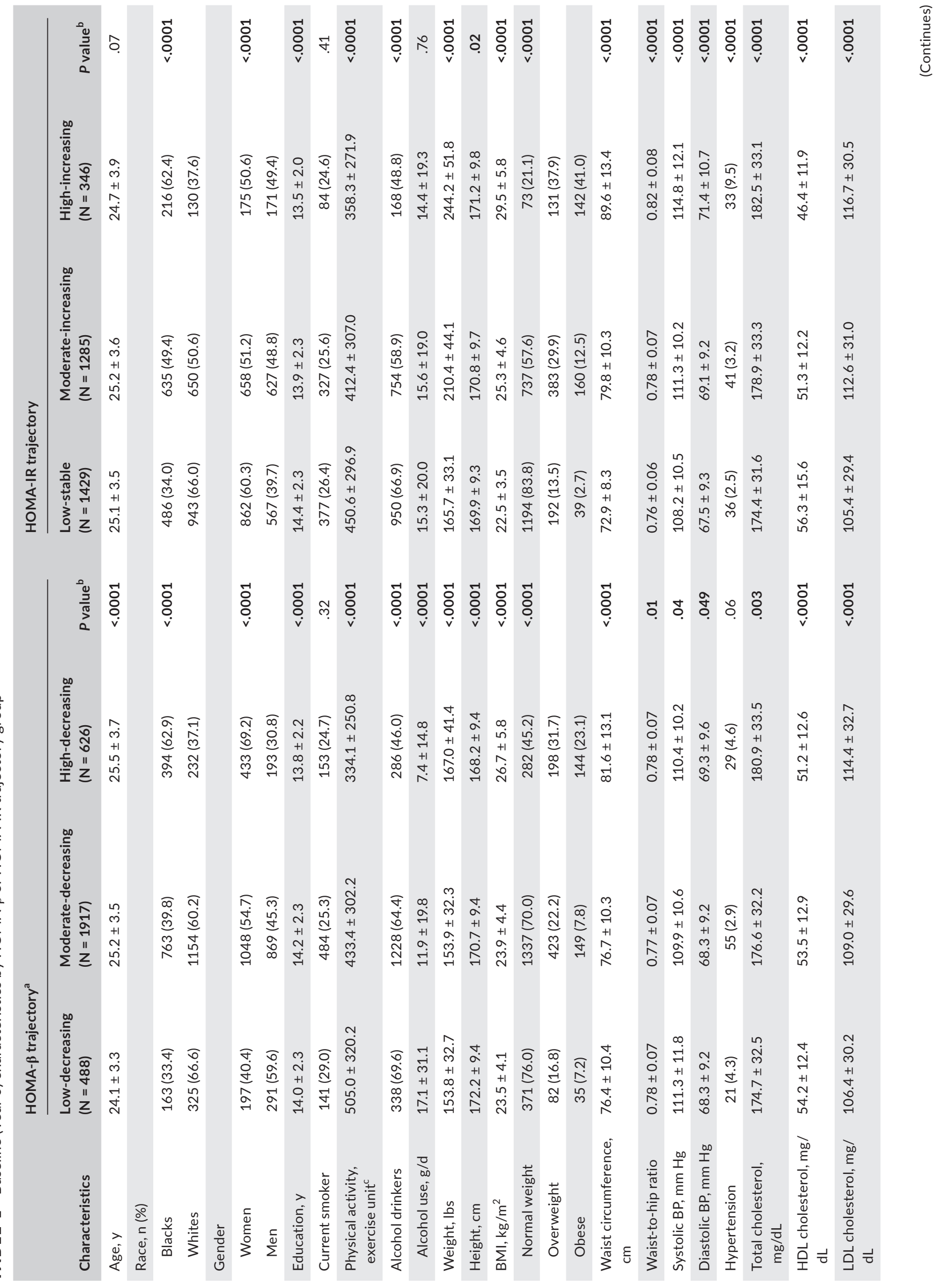




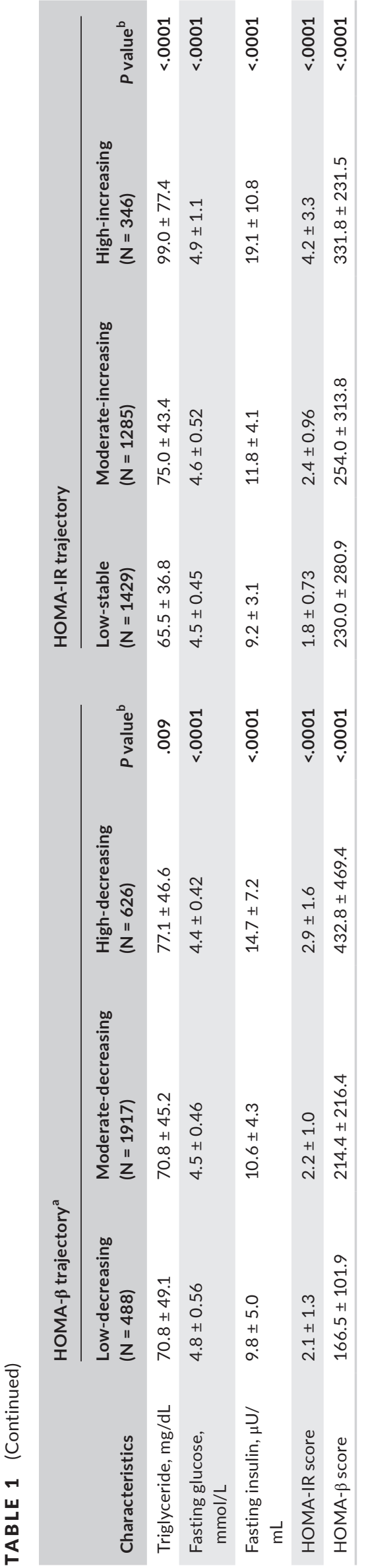

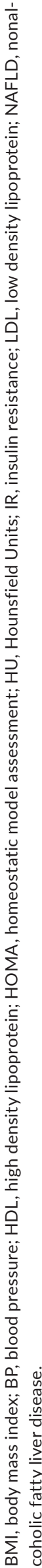

$<1)^{16}$ and moderate-severe NAFLD as a LA $\leq 40 \mathrm{HU}$ (equivalent to $\geq 30 \%$ liver fat) after exclusion of other liver fat causes. ${ }^{15}$

\section{3 | Statistical analysis}

Group-based trajectory modelling was used to identify and categorize participants based on patterns of longitudinal change in HOMA-IR or HOMA- $\beta$ during the 25 years of follow-up ( $N=3060$ for HOMA-IR, $N=3031$ for HOMA- $\beta$ ). ${ }^{17}$ HOMA-IR and HOMA- $\beta$ were logarithmically transformed to approximate normality. Models were fit using SAS Proc traj. ${ }^{18}$ Group-based trajectory analysis is designed to identify clusters of individuals with similar patterns of change over time. The optimal number of trajectory classes was determined using the Bayesian information criterion such that no group included less than $5 \%$ of participants. Participants were assigned to the trajectory group for which they had the greatest posterior predictive probability. ${ }^{17}$ Trajectory groups were then qualitatively examined and named to describe the visual pattern of change. To estimate the association of trajectory group with prevalent NAFLD or T2DM in NAFLD, trajectory group was included as an independent variable in a logistic regression model examining predictors of continuous LA or any NAFLD or moderate-severe NAFLD or T2DM in NAFLD at Y25. Models were sequentially adjusted a priori for demographics (age, gender, race, education, centre), cumulative burden of metabolic risk factors (pack-years of smoking, physical activity [exercise units per year], alcohol use [drinks/week], systolic blood pressure [SBP], total/high density lipoprotein cholesterol [HDL-C] ratio and number of visits with blood pressure or lipid-lowering medications), percent change in BMI relative to baseline, and HOMA-IR or HOMA- $\beta$ at YO or Y25. Cumulative SBP, alcoholic beverages, physical activity and total-HDL-C ratio were calculated by summing the product of the average SBP (alcohol or physical activity or $\mathrm{BMI}$ or total/HDL-C ratio) and the time interval (in years) between two consecutive examinations over the 25 years. To account for hypoglycaemic medication use over time, T2DM medication was included in the final model (Model 3) as a dichotomous variable at each exam year. Sensitivity analysis was also performed excluding measures from participants on any T2DM medications at any exam year. Interaction terms were assessed between trajectory group membership and race and sex. In addition, as a comparator group we assessed the relationship between HOMA-IR or HOMA- $\beta$ trajectory groups and prevalent/incident T2DM among the 1593 NAFLD-eligible participants with CT liver attenuation $>51 \mathrm{HU}$ (eg, non-NAFLD) in CARDIA (Figure S1).

Finally, three mutually exclusive clusters were defined based on hypothesized $\beta$-cell response and IR dynamics using observed HOMA-IR and HOMA- $\beta$ trajectory group membership pair (eg, increasing IR promotes $\beta$-cell demise and inhibits $\beta$-cell compensation, Table S2). Adjusting for the same set of covariates, logistic regression analyses were used to model the odds of having NAFLD or prevalent/incident T2DM among NAFLD participants at Y25 for each cluster in separate models. All analyses were completed using 


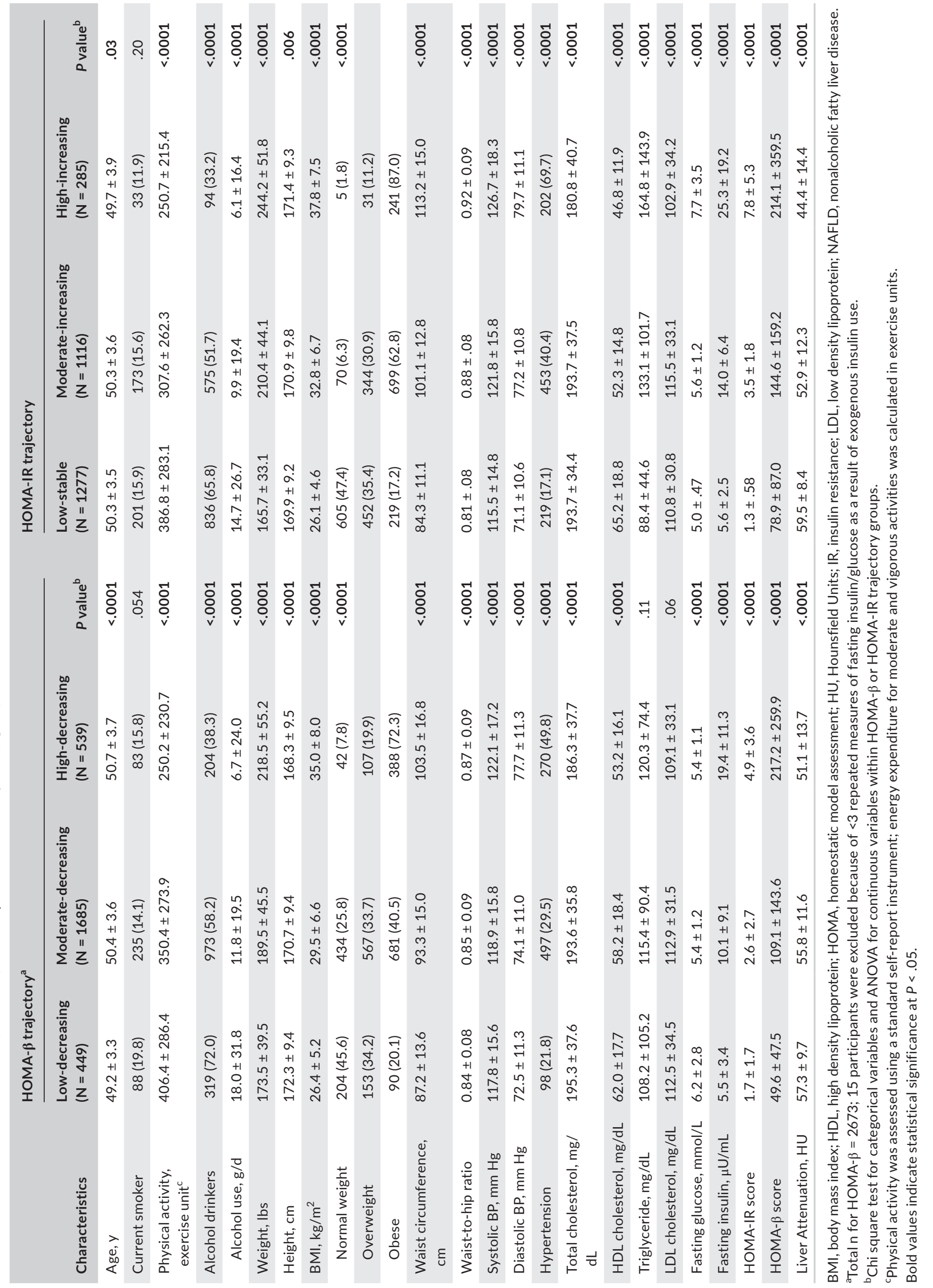


SAS software version 9.4 (SAS Institute Inc., Chicago, IL). Two-sided $P<.05$ was considered statistically significant.

\section{3 | RESULTS}

\subsection{HOMA-IR and HOMA- $\beta$ trajectories}

Three discrete trajectories in HOMA-IR and HOMA- $\beta$ from young adulthood to middle age were identified (Figure 1). Both HOMA-IR (Figure $1 \mathrm{~A}$ ) and HOMA- $\beta$ (Figure 1B) tracked over time among participants. For IR, nearly half of the cohort ( $n=1429,47 \%$ ) maintained fairly stable low IR throughout follow-up (low-stable) whereas $42 \%$ ( $n=1285$ ) had moderate increase in IR (moderate-increasing) and $12 \%(n=346)$ had high increase in IR (high-increasing). For HOMA- $\beta$, all trajectory groups demonstrated a decrease in HOMA- $\beta$ after age 45 (Figure 1B). However, the majority of participants maintained fairly HOMA- $\beta$ throughout most of young adulthood: $21 \%$ $(n=626)$ had high-decreasing HOMA- $\beta$ and 63\% ( $n=1917)$ had moderate-decreasing HOMA- $\beta$. Only $16 \%(n=488)$ of participants demonstrated a notable early and sustained decrease in HOMA- $\beta$ throughout young adulthood (eg, low-decreasing).

Participant characteristics at $\mathrm{YO}$ according to HOMA trajectory group are presented in Table 1. Individuals with highdecreasing HOMA- $\beta$ were older and predominantly women and black. Individuals with high-decreasing HOMA- $\beta$ were more likely to be overweight or obese compared to individuals with moderatedecreasing and low-decreasing HOMA- $\beta$. Similarly, individuals with high-increasing HOMA-IR were predominantly women, black and had higher baseline BMI compared to the low-stable IR group. At Y25, participants with moderate-decreasing and high-decreasing HOMA- $\beta$ were more likely to display features of the metabolic syndrome compared to participants in the low-decreasing group (Table 2). Similar trends were seen in participants with moderateincreasing or high-increasing IR throughout adulthood compared to participants with low-stable IR (Table 2).

\subsection{HOMA trajectories and NAFLD}

Non-alcoholic fatty liver disease prevalence in CARDIA was $24.5 \%$ and was higher with increasing HOMA-IR group. Any NAFLD (eg, LA $<51 \mathrm{HU}$ ) was present in $7.4 \%, 32.5 \%$, and $63.6 \%$ in the low-stable, moderate-increasing and high-increasing HOMA-IR groups respectively ( $P$ for trend $<.0001$, Figure $2 A$ ). Within each HOMA-IR group, NAFLD was greater in whites compared to blacks and in men compared to women, with the exception of the highincreasing HOMA-IR group in which white women had the highest NAFLD prevalence (Figure S2A). There was no significant interaction by race or gender in all models and thus pooled results are shown.

Table 3 demonstrates the association between HOMA-IR or HOMA- $\beta$ trajectory group and continuous LA or NAFLD. For HOMA-IR, those in trajectory groups with patterns of increasingly severe IR (referent: low-stable) had progressively greater odds of having any NAFLD after adjustment for demographic characteristics and education (Table 3 ). These associations were moderately attenuated when adjusted for demographics, cumulative burden of metabolic risk factors and YO HOMA-IR. Associations were attenuated more substantially, but remained statistically significant when adjusted for Y25 HOMA-IR. The association between HOMA-IR trajectory group and moderate-severe NAFLD (n events $=224$ ) was similar in direction and magnitude (Table 3). Findings were also similar for continuous LA.

Non-alcoholic fatty liver disease prevalence at Y25 was also higher with increasing HOMA- $\beta$ group, with NAFLD present in $14.3 \%, 21.3 \%$ and $40.8 \%$ of individuals in the low-decreasing, moderate-decreasing and high-decreasing groups respectively ( $P$ for trend $<.0001$, Figure $2 \mathrm{~B}$ ). NAFLD prevalence increased with increasing HOMA- $\beta$ trajectory in all race, gender groups with the exception of black women where NAFLD prevalence was $24.5 \%, 13.6 \%$ and $29.3 \%$ in the low, moderate and high HOMA- $\beta$ trajectory groups respectively (Figure S2B).

In comparison with individuals in the low-decreasing group, those in trajectory groups with higher HOMA- $\beta$ had progressively greater odds of NAFLD even after adjustment for demographics (Table 3). These associations were moderately attenuated when adjusted for

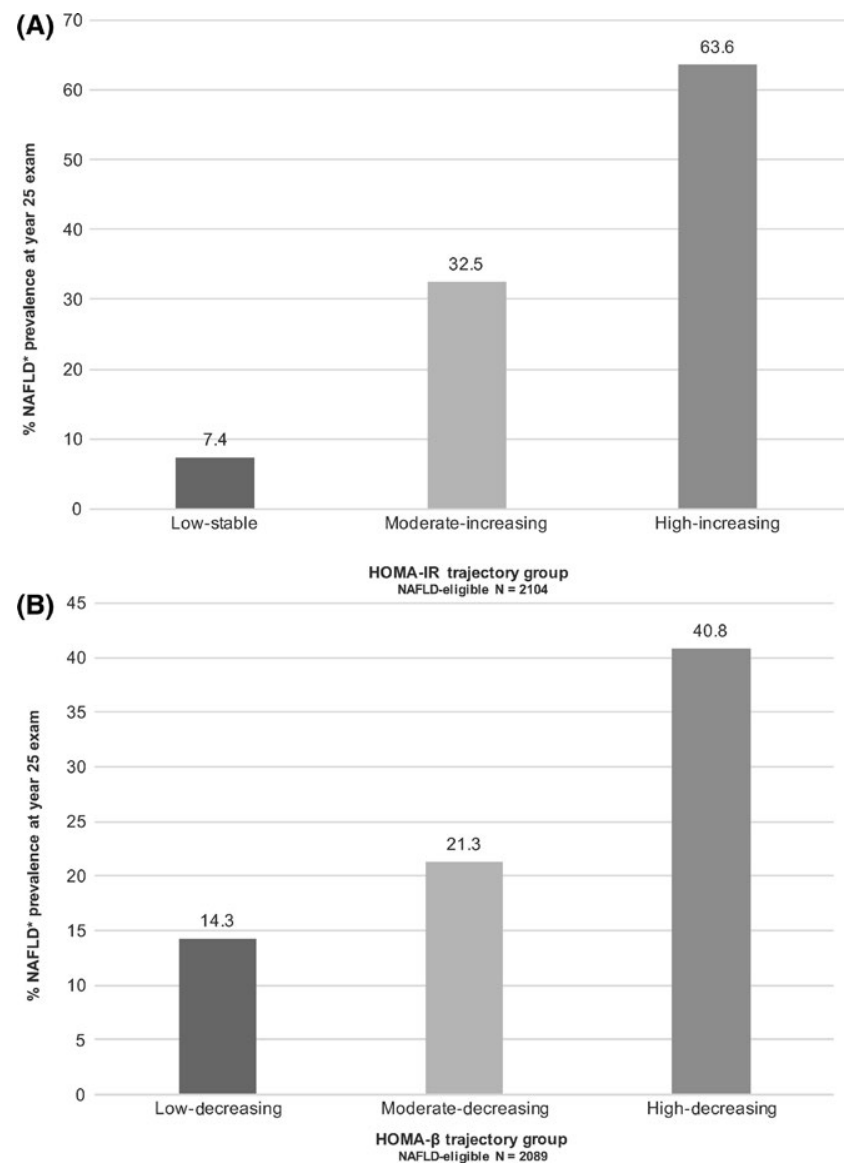

FIGURE 2 Year 25 NAFLD* Prevalence stratified by A, HOMA-IR and B, HOMA- $\beta$ Trajectory Group. Chi-square $P<.0001$ for trajectory group membership for both HOMA assessments. *NAFLD defined as CT liver attenuation $<51 \mathrm{HU}$ after exclusions for other causes of liver fat 


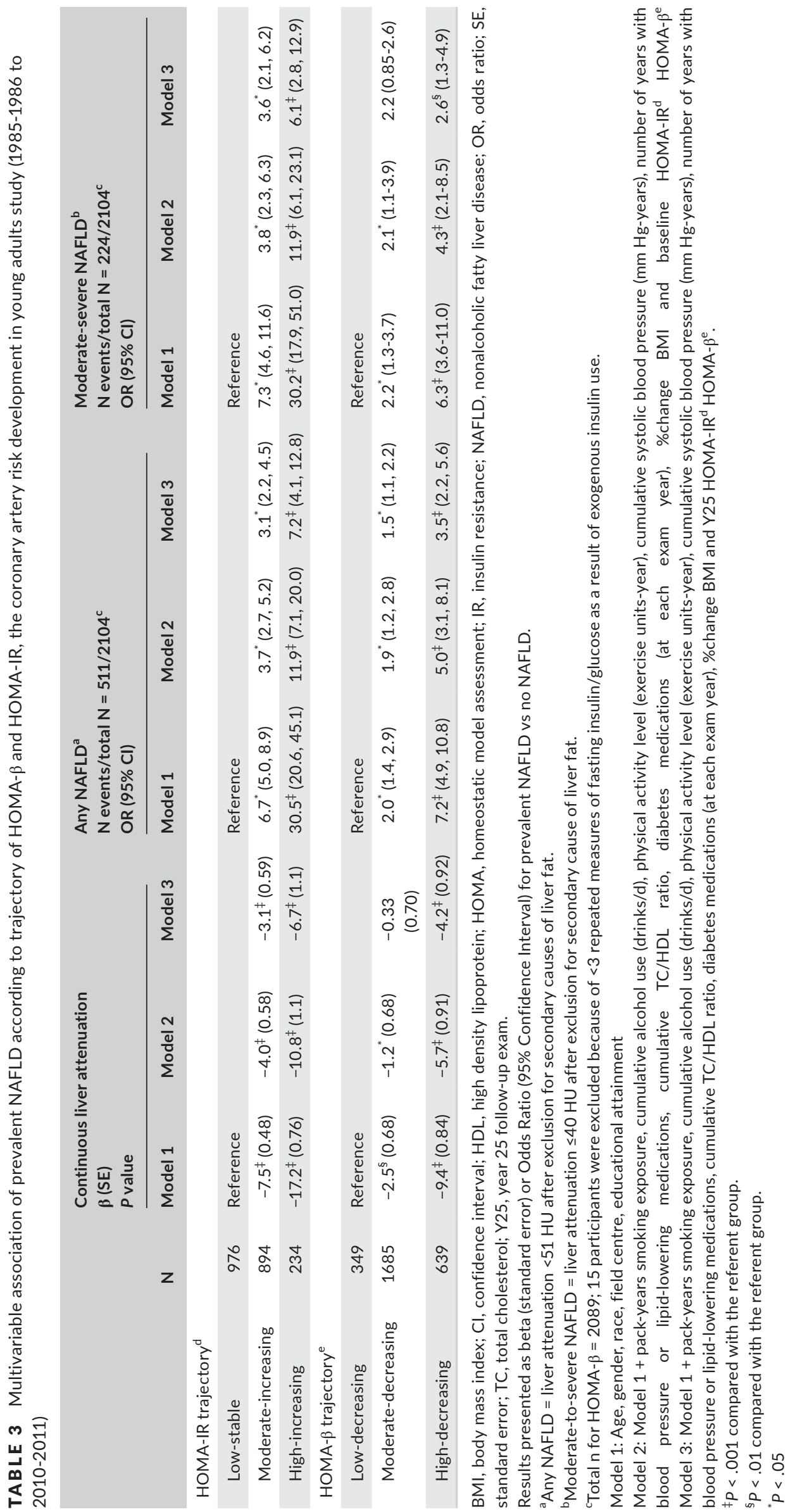


cumulative NAFLD risk factors, including YO HOMA- $\beta$. Associations were attenuated more substantially, but remained statistically significant when adjusted for Y25 HOMA- $\beta$. The association between HOMA- $\beta$ trajectory and continuous LA and moderate-severe NAFLD ( $n$ events $=224$ ) was similar in direction and magnitude (Table 3). In sensitivity analyses excluding those participants who were taking T2DM medications at any time point, all associations were unchanged (data not shown).

\subsection{HOMA trajectories and T2DM among NAFLD participants}

Among participants with any NAFLD $(n=511)$, both prevalent and incident T2DM at Y25 was higher with increasing HOMA-IR $(P<.0001$, Figure 3A). In contrast, participants with low-decreasing HOMA- $\beta$ trajectory had the highest prevalence of T2DM $(P<.0001$, Figure 3B). In multivariable analysis, low-decreasing HOMA- $\beta$ (referent: high-decreasing) trajectory was associated with higher odds of prevalent $(\mathrm{OR}=11.8[4.3,32.4])$ and incident $(\mathrm{OR}=9.1[3.4$, 24.3]) T2DM independent of cumulative T2DM risk factors and Y25 HOMA-IR (Table 4). In contrast, high-increasing HOMA-IR (referent: low-stable) trajectory was associated with greater prevalent $(\mathrm{OR}=4.6[1.1,18.9])$ and incident $(\mathrm{OR}=7.2[1.8,29.2]) \mathrm{T} 2 \mathrm{DM}$ (Table 4). Findings were similar in direction though somewhat stronger in magnitude in both unadjusted and adjusted analyses among non-NAFLD CARDIA participants who were excluded from the primary analyses $(n=1593$, Table S1).

\subsection{HOMA-IR and HOMA- $\beta$ trajectory group clusters}

The prevalence of CARDIA participants in each HOMA-IR/HOMA- $\beta$ trajectory group cluster are shown in Table S2. The low HOMA IR-low HOMA $\beta$ cluster and high HOMA IR-high HOMA $\beta$ cluster contained $45.7 \%$ and $21.1 \%$ of participants respectively. The remaining participants formed the referent cluster ( $n=1008,33.2 \%$ ). Participants in the high HOMA IR-high HOMA $\beta$ cluster were predominantly black (61.4\%) and women (63.8\%), and participants with low HOMA IRlow HOMA $\beta$ cluster were predominantly white (67.2\%) and women (57.5\%). Notably, participants in the high HOMA IR-high HOMA $\beta$ cluster had the lowest education and physical activity level, and less favourable levels for components of the metabolic syndrome at YO (Table S3) and at Y25 (Table S4).

Table 5 displays the odds of NAFLD or T2DM among NAFLD participants at $\mathrm{Y} 25$ for the 3 HOMA IR-HOMA $\beta$ trajectory group clusters. The odds of NAFLD were significantly higher in the high HOMA IR-high HOMA $\beta$ cluster than the referent cluster in all models. When IR and $\beta$-cell response trajectories were both high, the odds of developing NAFLD were significantly higher than the referent. In contrast, participants in the low HOMA IR-low HOMA $\beta$ cluster had significantly lower odds of NAFLD compared to the referent. In terms of T2DM risk, NAFLD participants in the high HOMA IR-high HOMA $\beta$ cluster had significantly higher odds of
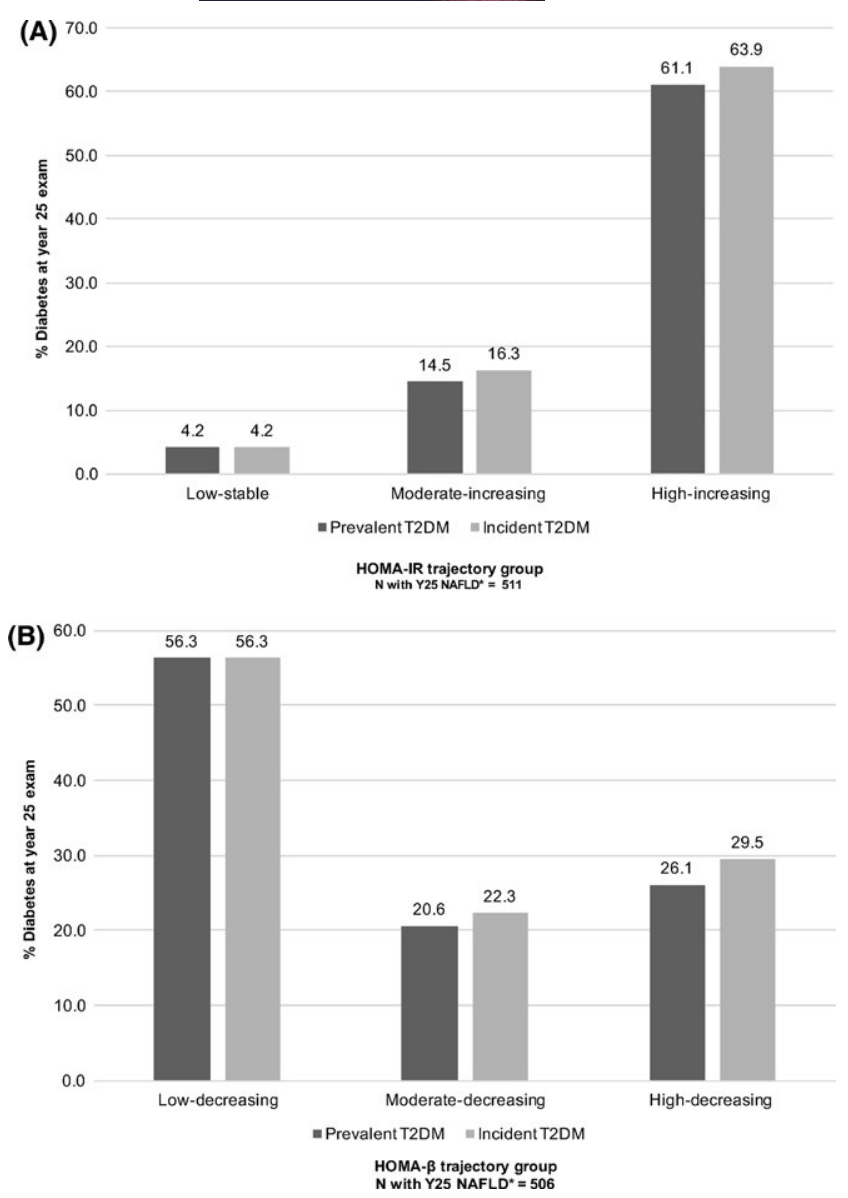

FIGURE 3 Year 25 Prevalent and Incident Diabetes among persons with NAFLD* stratified by A, HOMA-IR and B, HOMA- $\beta$ Trajectory Group. Chi-square $P<.0001$ for trajectory group membership for both HOMA assessments. *NAFLD defined as CT liver attenuation $<51 \mathrm{HU}$ after exclusions for other causes of liver fat. Estimates are for participants with diabetes by $\mathrm{Y} 25$ follow-up, not at $Y 25$ follow-up, thus incidence is >prevalence

both prevalent and incident T2DM in the base model, but associations were attenuated and no longer significant in the fully adjusted model.

\section{4 | DISCUSSION}

In this population-based prospective study of black and white adults followed up for 25 years, we identified three distinct trajectories of IR and pancreatic $\beta$-cell response to glucose, separately. These trajectories were independently associated with prevalent NAFLD and T2DM among individuals with NAFLD in midlife. We found that those groups with greater IR increase from young adulthood into middle age have the greatest odds of having NAFLD and subsequent T2DM, regardless of demographics, cumulative burden of metabolic covariates, and degree of IR at YO or concurrently at Y25. Higher $\beta$-cell response, was also related to higher odds of NAFLD, however, lower $\beta$-cell response, was related to higher odds of T2DM. When taken 
TAB LE 4 Prevalent or incident type 2 diabetes mellitus according to HOMA- $\beta$ or HOMA-IR trajectory group among 511 participants with prevalent NAFLD at the year 25 exam, the coronary artery risk development in young adults study (1985-1986 to 2010-2011)

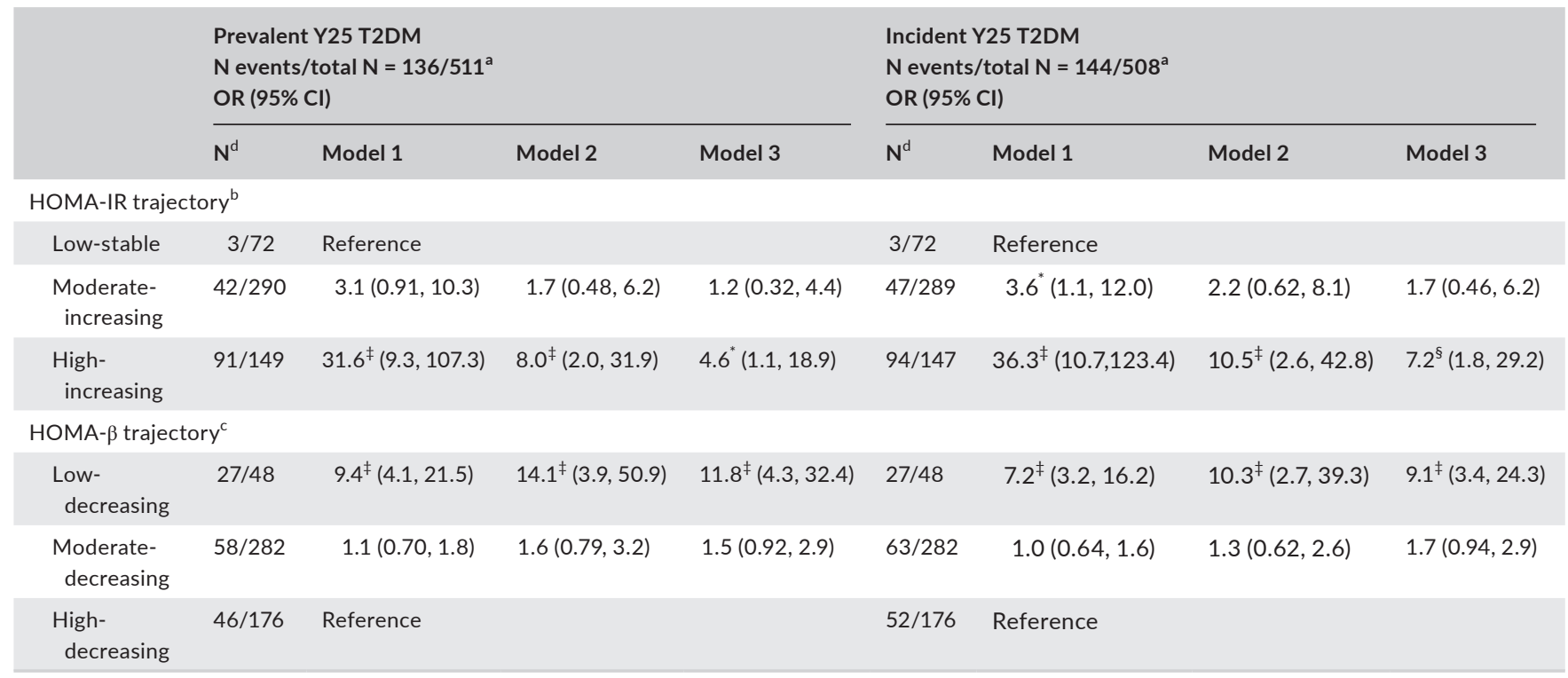

BMI, body mass index; $\mathrm{Cl}$, confidence interval; HDL, high density lipoprotein; HOMA, homeostatic model assessment; IR, insulin resistance; NAFLD, nonalcoholic fatty liver disease; OR, odds ratio; T2DM, type 2 diabetes mellitus; TC, total cholesterol; Y25, year 25 follow-up exam.

NAFLD = liver attenuation $<51 \mathrm{HU}$ after exclusion for secondary cause of liver fat.

Results presented as Odds Ratio (95\% Confidence Interval).

${ }^{\text {aTotal }} \mathrm{N}=506$ for analyses with HOMA- $\beta$ after exclusion of participants with exogenous insulin use.

Model 1: Age, gender, race, field center, educational attainment Model 2: Model $1+$ pack-years smoking exposure, cumulative alcohol use (drinks/d), physical activity level (exercise units-year), cumulative systolic blood pressure (mm Hg-years), number of years with blood pressure or lipid-lowering medications, cumulative TC/HDL ratio, diabetes medications (at each exam year), \%change BMI and baseline HOMA-IR ${ }^{b}$ HOMA- $\beta^{c}$ Model 3: Model $1+$ pack-years smoking exposure, cumulative alcohol use (drinks/d), physical activity level (exercise units-year), cumulative systolicblood pressure (mm Hg-years), number of years with blood pressure or lipid-lowering medications, cumulative TC/HDL ratio, diabetes medications (at each exam year), \%change BMI and Y25 HOMA-IR ${ }^{\mathrm{b}} \mathrm{HOMA}-\beta^{\mathrm{c}}$.

${ }^{\mathrm{d}}$ Number of participants with diabetes over the total number of NAFLD participants in each trajectory group.

${ }^{\ddagger} P<.001$ compared with the referent group.

${ }^{\S} P<.01$ compared with the referent group.

${ }^{*} P<.05$ compared to Low group.

collectively, persons with high $\beta$-cell response, in relation to high IR had the highest odds of prevalent NAFLD and T2DM in midlife independent of cumulative metabolic confounders. These findings highlight early identification of increasing IR and pancreatic $\beta$-cell response to glucose, as potential targets for primary prevention of NAFLD and T2DM in NAFLD.

Multiple studies have demonstrated hyperinsulinaemia, and thus elevated HOMA-IR, in NAFLD. ${ }^{19}$ IR is inadequate response by tissues to the physiological effects of insulin. IR is thus tissue-specific. HOMA-IR typically reflects hepatic IR. ${ }^{20}$ However, the liver is also the primary site of insulin clearance in humans. ${ }^{21}$ Several studies have shown that HOMA-IR elevation in NAFLD is primarily related to impaired hepatic insulin clearance rather than impaired insulin suppression of hepatic glucose production. ${ }^{1,22}$ Thus, HOMA-IR may be a poor overall marker of hepatic IR in NAFLD and the relationship between glucose and insulin is impaired in these patients. In contrast, adipose IR may be an important driver of NAFLD pathogenesis: dysfunctional adipose tissue $\rightarrow$ increase in circulating free fatty acids $\rightarrow$ hepatic steatosis (eg, lipotoxicity). ${ }^{22,23}$ Therefore, in NAFLD, HOMA-IR may be a risk marker for underlying dysfunctional adipose tissue and may not necessarily correlate with hepatic IR. Direct measures of adipose tissue IR would add strength to our study but are not available.

We also demonstrate that high pancreatic $\beta$-cell response to glucose throughout adulthood is a marker of NAFLD independent of metabolic risk factors. Previous studies likewise demonstrate that liver fat is associated with absolute increases in insulin secretion from the $\beta$-cell, in order to compensate for IR and maintain euglycemia. ${ }^{24}$ It has also been demonstrated that NAFLD is associated with pancreatic $\beta$-cell dysfunction in non-diabetic obese subjects. ${ }^{8,9}$ NAFLD individuals have an exaggerated $\beta$-cell insulin secretory response to an oral glucose load independent of BMI, age and sex; and a decline in $\beta$-cell index, which reflects pancreatic $\beta$-cell function, in the setting of underlying IR. ${ }^{8,23}$ Our current findings add to the epidemiological evidence that increasing IR and pancreatic $\beta$-cell response to glucose are markers of underlying metabolic disarray that predisposes to risk for T2DM in persons with both NAFLD and non-NAFLD. However, it is important to note that similar to HOMA-IR, HOMA- $\beta$ is also affected by degree of hepatic insulin clearance, which is proportional to hepatic 
TABLE 5 Relationship between HOMA- $\beta$ and HOMA-IR trajectory group clusters in relation to risk of $\mathrm{Y} 25 \mathrm{NAFLD}^{\mathrm{a}}$ and prevalent or incident type 2 diabetes among NAFLD participants

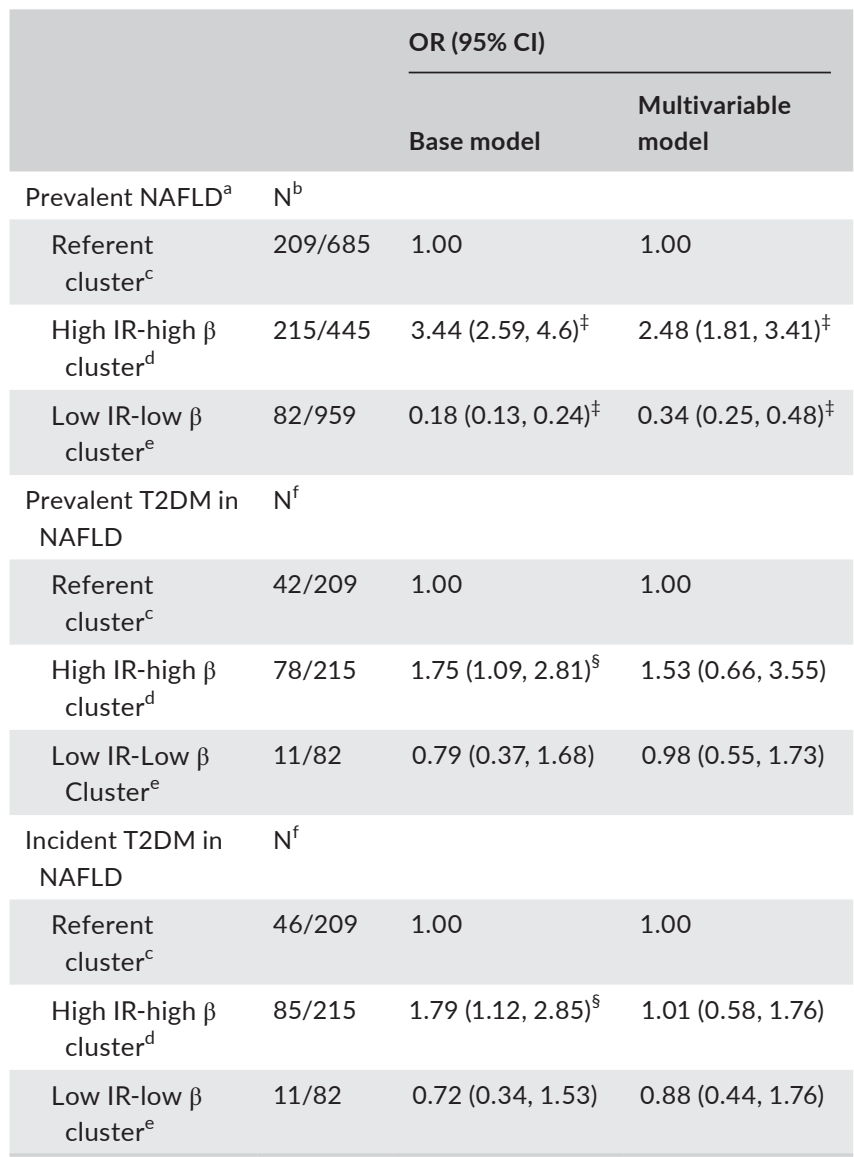

$\mathrm{BMI}$, body mass index; $\mathrm{Cl}$, confidence interval; $\mathrm{HDL}$, high density lipoprotein; HOMA, homeostatic model assessment; IR, insulin resistance; NAFLD, nonalcoholic fatty liver disease; OR, odds ratio; T2DM, type 2 diabetes mellitus; TC, total cholesterol; Y25, year 25 follow-up exam. Base model: Age, gender, race, field center, educational attainment. Multivariable Model: Base model + pack-years smoking exposure, cumulative alcohol use (drinks/d), physical activity level (exercise units-year), cumulative systolic blood pressure (mm Hg-years), number of years with blood pressure or lipid-lowering medications, cumulative TC/HDL ratio, diabetes medications (at each exam year), \%change BMI, Y25 HOMA-IR and Y 25 HOMA- $\beta$.

${ }^{\mathrm{a}} \mathrm{NAFLD}=$ liver attenuation $<51 \mathrm{HU}$ after exclusion for secondary cause of liver fat.

${ }^{\mathrm{b}}$ Number of participants with NAFLD over the total number of NAFLDeligible participants $(n=2089)$ assessed at the year 25 follow-up exam in each trajectory group pair.

${ }^{c}$ Referent Cluster $=$ high $\mathrm{HOMA}-\mathrm{IR}$ and low HOMA- $\beta$, moderate HOMA-IR and moderate HOMA- $\beta$ or low HOMA-IR and high HOMA- $\beta$. ${ }^{\mathrm{d}}$ High IR-High $\beta$ cluster $=$ high HOMA-IR and high HOMA- $\beta$, high HOMA-IR and moderate HOMA- $\beta$ or moderate HOMA-IR and high HOMA- $\beta$.

${ }^{e}$ Low IR-Low $\beta$ cluster = low HOMA IR and low HOMA- $\beta$, low HOMA-IR and moderate HOMA- $\beta$, or moderate HOMA-IR and low HOMA- $\beta$.

${ }^{f}$ Number of participants with T2DM over the total number of NAFLD participants $(n=506)$ at the year 25 follow-up exam in each trajectory group pair.

${ }^{\ddagger} P<.001$ compared with the referent group.

${ }^{\S} P<.01$ compared with the referent group.

fat content. Thus, HOMA- $\beta$ may be a poor overall marker of pancreatic $\beta$-cell function in NAFLD. Prospective studies that assess rate of change in hepatic insulin clearance over time are needed to fully understand the role of IR and pancreatic $\beta$-cell response during NAFLD development.

Progression to diabetes is a complex interplay among IR, insulin sensitivity, and pancreatic $\beta$-cell function. A decrease in sensitivity to insulin demands compensation through a proportionate adjustment in insulin secretion by pancreatic $\beta$-cells to maintain glucose homeostasis. ${ }^{25}$ On a population-level, T2DM risk is driven by multiple factors. We have now demonstrated among persons with both NAFLD and non-NAFLD that increasing level of IR over time is an important driver in the risk of subsequent T2DM. In addition, we found that high IR and high $\beta$-cell response in participants with NAFLD increased odds of incident T2DM by $30 \%$ compared to participants with NAFLD and high IR and low $\beta$-cell response. In contrast, low IR despite low pancreatic $\beta$-cell response was associated with a $36 \%$ decreased odds of T2DM suggesting that IR is the primary driver of T2DM in NAFLD. Once established, T2DM may promote progression to NASH, cirrhosis and hepatocellular carcinoma. ${ }^{2}$ Thus, improved understanding of risk factors for development of T2DM in NAFLD is critical in order to target prevention of liver disease progression.

Several limitations should also be considered when interpreting our results. CT is a relatively insensitive measure of hepatic fat when compared with magnetic resonance imaging, ${ }^{16,26}$ which may bias our results toward the null and underestimate the strength of the observed association. NAFLD was also not assessed in CARDIA prior to the Y25 follow up examination and thus, we cannot establish temporality of our HOMA-IR and HOMA- $\beta$ trajectories or incident diabetes in relation to NAFLD onset. However, since NAFLD is primarily an asymptomatic disease, detection in midlife mirrors clinical practice when NAFLD is commonly incidentally found on imaging performed for other reasons. ${ }^{27}$ It is also possible that some CARDIA participants had undiagnosed NAFLD at Y0. However, 62\% of NAFLD participants were normal weight at $Y 0$ and only $10 \%$ had obesity. Since undiagnosed NAFLD may have been present early in follow up, we also cannot rule out the possibility of reverse causality (eg, NAFLD causing an increase in IR, rather than vice versa). CARDIA only included whites and blacks and did not specifically ask about ethnicity. Thus, we cannot generalize our findings to the Hispanic population wherein NAFLD and T2DM are exceedingly high. The assays for glucose and insulin changed during the 25-year follow-up because of technological advances. However, $83 \%$ of the measurements were assessed using the same method. Finally, we employed surrogate markers of IR and $\beta$-cell response and did not use repeated OGTT or frequent sampling of intravenous glucose tolerance test or c-peptide levels to measure IR or pancreatic $\beta$ cell function. Thus, we cannot directly relate insulin resistance to insulin secretion within an individual. We are also unable to assess effect of degree of hepatic insulin clearance on absolute levels of HOMA-IR or HOMA- $\beta$. However, HOMA models can be easily calculated in clinical practice and thus temporal trends (rather than absolute levels) may be useful for detection of NAFLD individuals at high risk for T2DM. 


\section{1 | Potential clinical implications}

Higher IR is a well-known risk factor for the development of NAFLD and T2DM and $\beta$-cell failure is a known requirement for the development of T2DM in IR patients. However, our findings suggest than an individual's long-term pattern of change in insulin sensitivity and secretion starting in early adulthood provides additional information about their risk for the development of NAFLD and T2DM in midlife independent of absolute level of insulin resistance or $\beta$-cell response in early adulthood or in midlife. In the age of the electronic medical record, repeated measures of insulin sensitivity and secretion throughout adulthood can be readily graphed allowing clinicians to recognize at-risk patterns (eg, rapidly rising IR) early in adulthood. Early identification of young adults with high HOMA-IR trajectory may lead to treatments that target prevention of adipose tissue overload (eg, weight maintenance, physical activity) and prevention of adipose tissue insulin signalling inactivation (eg, pioglitazone) for primary prevention of NAFLD. The potential clinical implications of our findings require further prospective study.

\section{5 | CONCLUSION}

Trajectories of IR and pancreatic $\beta$-cell response to glucose concentration throughout early adulthood to middle age-independent of baseline and concurrent IR and $\beta$-cell response-may provide additional information about the cumulative burden of IR and risk of prevalent NAFLD and T2DM in midlife. These associations were independent of key comorbidities and metabolic risk factors. This novel characterization of the relationship between IR and $\beta$-cell response trajectories across young adulthood highlights this age period as an important time to target behaviour and lifestyle interventions for primordial prevention of NAFLD and subsequent T2DM.

\section{ACKNOWLEDGEMENTS}

The authors thank the CARDIA participants for their long-term commitment to the study.

\section{CONFLICTS OF INTEREST}

The authors do not have any disclosures to report.

\section{ORCID}

Lisa B. VanWagner (iD http://orcid.org/0000-0002-6264-2573

Miriam B. Vos iD http://orcid.org/0000-0002-0817-7068

\section{REFERENCES}

1. Gaggini M, Morelli M, Buzzigoli E, DeFronzo RA, Bugianesi E, Gastaldelli A. Non-alcoholic fatty liver disease (NAFLD) and its connection with insulin resistance, dyslipidemia, atherosclerosis and coronary heart disease. Nutrients. 2013;5:1544-1560.

2. Anstee QM, Targher G, Day CP. Progression of NAFLD to diabetes mellitus, cardiovascular disease or cirrhosis. Nat Rev Gastroenterol Hepatol. 2013;10:330-344.

3. Byrne CD, Targher G. NAFLD: a multisystem disease. J Hepatol. 2015;62(1 Suppl):S47-S64.

4. Marchesini G, Marzocchi R. Metabolic syndrome and NASH. Clin Liver Dis. 2007;11:105-117, ix.

5. Rakoski MO, Singal AG, Rogers MA, Conjeevaram H. Meta-analysis: insulin sensitizers for the treatment of non-alcoholic steatohepatitis. Aliment Pharmacol Ther. 2010;32:1211-1221.

6. Mazzotti A, Caletti MT, Marchignoli F, Forlani G, Marchesini G. Which treatment for type 2 diabetes associated with non-alcoholic fatty liver disease? Dig Liver Dis. 2017;49:235-240.

7. European Association for the Study of the Liver (EASL), European Association for the Study of Diabetes (EASD), European Association for the Study of Obesity (EASO). EASL-EASD-EASO Clinical Practice Guidelines for the management of non-alcoholic fatty liver disease. J Hepatol. 2016;64:1388-1402.

8. Siddiqui MS, Cheang KL, Luketic VA, et al. Nonalcoholic Steatohepatitis (NASH) Is Associated with a Decline in Pancreatic Beta Cell (beta-Cell) Function. Dig Dis Sci. 2015;60:2529-2537.

9. Bedogni G, Gastaldelli A, Tiribelli C, et al. Relationship between glucose metabolism and non-alcoholic fatty liver disease severity in morbidly obese women. J Endocrinol Invest. 2014;37:739-744.

10. Rijkelijkhuizen JM, Doesburg T, Girman CJ, et al. Hepatic fat is not associated with beta-cell function or postprandial free fatty acid response. Metabolism. 2009;58:196-203.

11. Wallace TM, Levy JC, Matthews DR. Use and abuse of HOMA modeling. Diabetes Care. 2004;27:1487-1495.

12. Friedman GD, Cutter GR, Donahue RP, et al. CARDIA: study design, recruitment, and some characteristics of the examined subjects. J Clin Epidemiol. 1988;41:1105-1116.

13. Bergman RN, Ader M, Huecking K, Van Citters G. Accurate assessment of beta-cell function: the hyperbolic correction. Diabetes. 2002;51(Suppl 1):S212-S220

14. Parker ED, Schmitz KH, Jacobs DR, Dengel DR, Schreiner PJ. Physical activity in young adults and incident hypertension over 15 years of follow-up: the CARDIA study. Am J Public Health. 2007;97:703-709.

15. VanWagner LB, Ning $H$, Lewis CE, et al. Associations between nonalcoholic fatty liver disease and subclinical atherosclerosis in middle-aged adults: The Coronary Artery Risk Development in Young Adults Study. Atherosclerosis. 2014;235:599-605.

16. Kodama $\mathrm{Y}, \mathrm{Ng} \mathrm{CS}, \mathrm{Wu} \mathrm{TT}$, et al. Comparison of CT methods for determining the fat content of the liver. AJR Am J Roentgenol. 2007;188:1307-1312.

17. Allen NB, Siddique J, Wilkins JT, et al. Blood pressure trajectories in early adulthood and subclinical atherosclerosis in middle age. JAMA. 2014;311:490-497.

18. Nagin DS, Odgers CL. Group-based trajectory modeling (nearly) two decades later. J Quant Criminol. 2010;26:445-453.

19. Gutierrez-Buey G, Nunez-Cordoba JM, Llavero-Valero M, Gargallo J, Salvador J, Escalada J. Is HOMA-IR a potential screening test for non-alcoholic fatty liver disease in adults with type 2 diabetes? Eur J Intern Med. 2017;41:74-78.

20. Tripathy D, Almgren P, Tuomi T, Groop L. Contribution of insulinstimulated glucose uptake and basal hepatic insulin sensitivity to surrogate measures of insulin sensitivity. Diabetes Care. 2004;27:2204-2210.

21. Duckworth WC, Kitabchi AE. Insulin metabolism and degradation. Endocr Rev. 1981;2:210-233. 
22. Bril F, Barb D, Portillo-Sanchez P, et al. Metabolic and histological implications of intrahepatic triglyceride content in nonalcoholic fatty liver disease. Hepatology. 2017;65:1132-1144.

23. Fabbrini E, Magkos F. Hepatic steatosis as a marker of metabolic dysfunction. Nutrients. 2015;7:4995-5019.

24. Pagano G, Pacini G, Musso G, et al. Nonalcoholic steatohepatitis, insulin resistance, and metabolic syndrome: further evidence for an etiologic association. Hepatology. 2002;35:367-372.

25. Kahn SE. The relative contributions of insulin resistance and beta-cell dysfunction to the pathophysiology of Type 2 diabetes. Diabetologia. 2003;46:3-19.

26. Musso G, Gambino R, Cassader M, Pagano G. Meta-analysis: natural history of non-alcoholic fatty liver disease (NAFLD) and diagnostic accuracy of non-invasive tests for liver disease severity. Ann Med. 2011;43:617-649.

27. Blais P, Husain N, Kramer JR, Kowalkowski M, El-Serag H, Kanwal F. Nonalcoholic fatty liver disease is underrecognized in the primary care setting. Am J Gastroenterol. 2015;110:10-14.

\section{SUPPORTING INFORMATION}

Additional Supporting Information may be found online in the supporting information tab for this article.

How to cite this article: VanWagner LB, Ning H, Allen NB, et al. Twenty-five-year trajectories of insulin resistance and pancreatic $\beta$-cell response and diabetes risk in nonalcoholic fatty liver disease. Liver Int. 2018;38:2069-2081.

https://doi.org/10.1111/liv.13747 